\section{Kompass \\ Ophthalmologie}

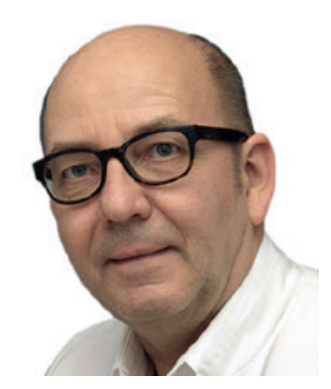

\title{
Uveitis on nivolumab: When it rains, it pours...
}

\author{
Uwe Pleyer \\ Klinik für Augenheilkunde, Charité - Universitätsmedizin Berlin, Campus Virchow-Klinikum, Berlin, Germany
}

Abstract of Obata S, Saishin Y, Teramura K, et al.: Vogt-Koyanagi-Harada disease-like uveitis during nivolumab (anti-PD-1 antibody) treatment for metastatic cutaneous malignant melanoma. Case Rep Ophthalmol 2019;10:67-74.

\section{Keywords}

Vogt-Koyanagi-Harada disease · Nivolumab · Malignant melanoma Programmed cell death protein 1 uveitis

\begin{abstract}
Nivolumab is an anti-programmed cell death protein 1 monoclonal antibody that is used to treat metastatic cutaneous malignant melanoma. Although bilateral uveitis has been reported as a side effect of nivolumab administration, there are few reports of VogtKoyanagi-Harada disease (VKH)-like uveitis. We report such a case. A 63-year-old woman with metastatic cutaneous malignant melanoma experienced visual loss in both eyes 10 days after her second nivolumab injection. Her decimal best-corrected visual acuity (BCVA) was 0.7 in the right eye and 0.4 in the left eye. Examination revealed bilateral granulomatous keratic precipitates and posterior synechiae in the left eye. Optical coherence tomography showed multiple sites of serous retinal detachment (SRD) in
\end{abstract}

the left eye and wavy retinal pigment epithelium in both eyes. Fluorescein angiography revealed multiple pinpoint-sized areas of leakage in both eyes and active leakage from the disc in her right eye. Indocyanine green angiography (IA) showed choroidal hyperfluorescence due to choroidal vascular leakage, with hypofluorescent dark spots during the late phase. These findings supported a diagnosis of VKH-like uveitis following nivolumab injections. Nivolumab was discontinued because of headache. Anterior chamber inflammation disappeared 3 weeks after starting topical corticosteroid treatment, and the SRD disappeared within 3 months. Her decimal BCVA recovered to $1.0 \mathrm{in}$ the right eye and to 0.9 in the left eye. Also, the fluorescein angiography and IA findings had improved by 4 months. We concluded that careful follow-up is required after nivolumab treatment because VKH-like panuveitis might develop.

C 2019 The Author(s). Published by S. Karger AG, Basel 


\section{Knowledge transfer}

\section{Background}

Nivolumab is a human, monoclonal antibody which binds to the programmed cell death 1 (PD-1) receptor and is used for the treatment of metastatic malignant melanoma of the skin. The binding of the PD-1 receptor to PD-L1 and PD-L2 leads to the inhibition of T-cell proliferation and increases its activity, including the tumour defence reaction (fig. 1). This enabled previously unknown therapeutic successes with a remission of the tumour and metastases and a significant prolongation of life achieved. At the same time, the patient's immunotolerance is changed and a number of side effects can occur. Although bilateral intraocular inflammation has been reported following the administration of nivolumab, there are only a few reports on uveitis similar to Vogt-Koyanagi-Harada (VKH) disease.

\section{Casuistry}

A 63-year-old woman who suffered from advanced, already metastatic malignant melanoma of the skin is presented. The primary lesion was surgically removed and then treated with nivolumab. 10 days after the second nivolumab injection, the patient suffered significant visual impairment in both eyes, to 0.7 in the right eye and 0.4 in the left eye. The characteristic image of a VKH manifestation could be confirmed clinically and through indocyanine green angiography (fig. 2). Due to the simultaneously existing severe headaches which are also typical of this syndrome, nivolumab was discontinued and anti-inflammatory treatment with steroids was initiated. During follow-up examinations, a largely morphological and functional remission could be observed within 3 months.

\section{Conclusion for clinical practice}

The immune-mediated side effects observed in the treatment of nivolumab are, among other things, ocular changes (apart from skin, lungs and intestines).

Intraocular inflammation is of the greatest significance here. Uveitis was reported in $0.4 \%$ of the patients in the framework of approval studies of PD-1 therapies [1]. It overwhelmingly occurred as anterior uveitis, which responds well to topical or intraocular steroids [24].

In the present casuistry, the typical changes of VKH syndrome occurred, with bilateral, diffuse, granulomatous uveitis and involvement of the central nervous system. The pathogenic mechanism of VKH syndrome is suspected to be a melanocyte-directed immunological disorder with T-cell-dependent cytotoxicity and apoptosis. Clinically, this occurred due to changes in the skin (poliosis and vitiligo), the nervous system (pleocytosis in the CSF with headaches, nausea and dizziness) and of the eye (choroid granuloma). Malignant melanoma cells and choroid melanocytes presumably have a common target epitope for the detection of T cells. In this way, the pathogenesis of VKH syndrome, which is attributed to an autoimmune reaction against melanocytes, is indirectly confirmed.

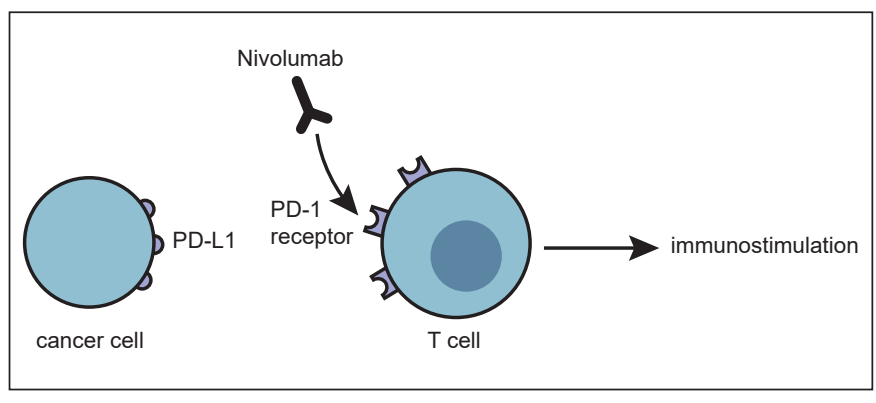

Fig. 1. Mechanism of action of nivolumab (modified according to www. pharmawiki.ch).
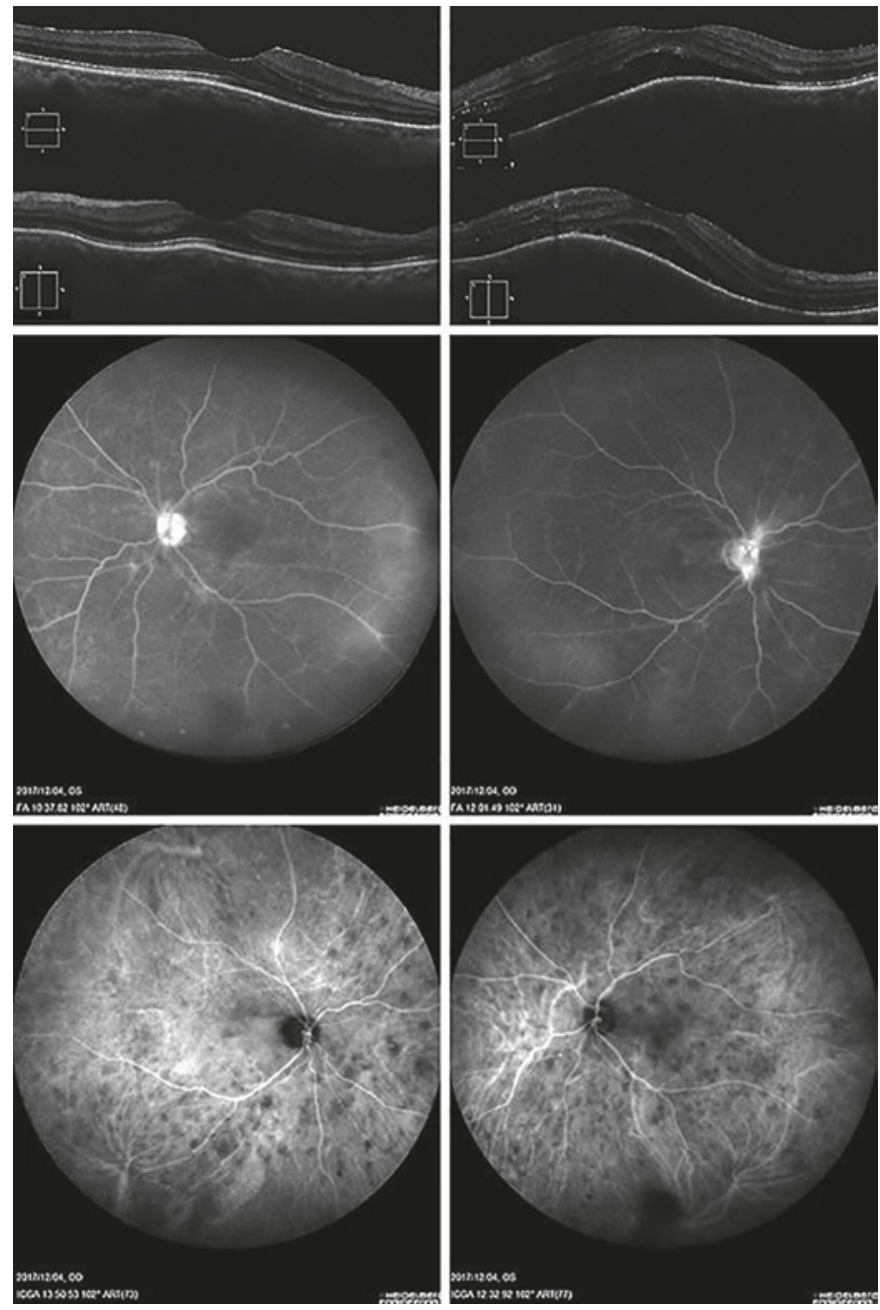

Fig. 2. Right eye (left) and left eye (right). Vertical scan of the optical coherence tomography prior to treatment. Several sites with serous retinal detachment as well as changes to the retinal pigment epithelium of both eyes can be seen. The fluorescein angiography prior to treatment shows multiple circumscribed leakages of both eyes as well as active leakage from the papilla of the right eye. The indocyanine green angiography additionally showed choroidal hyperfluorescence due to choroidal vascular leakage, followed by hyperfluorescent dark spots in the late phase. 
The development of a dry eye, in some cases with a severe course, has been reported as another nivolumab-associated side effect (phase I study in 2 out of 207 patients) [4, 5]. Because of the immune-mediated side effects, patients should be closely monitored (at least up to 5 months after the last dose) since side effects of nivolumab can occur at any time during or after treatment.

\section{Disclosure Statement}

There are no conflicts of interest with regard to this commentary.

\section{Republication}

This article was first published in Karger Kompass Ophthalmol 2019; 5:127-129.

\section{References}

1 Robert C, Schachter J, Long GV, et al.: Pembrolizumab versus ipilimumab in advanced melanoma. N Engl J Med 2015;372:2521-2532.

2 De Velasco G, Bermas B, Choueiri TK: Autoimmune arthropathy and uveitis as complications of programmed death 1 inhibitor treatment. Arthritis Rheumatol 2016;68:556-557.

3 Arai T, Harada K, Usui Y, et al.: Case of acute anterior uveitis and Vogt-Koyanagi-Harada syndrome-like eruptions induced by nivolumab in a melanoma patient. J Dermatol 2017;44:975-976.

4 Nguyen AT, Elia M, Materin MA, et al.: Cyclosporine for dry eye associated with nivolumab: A case progressing to corneal perforation. Cornea 2016;35: 399-401.

5 Brahmer JR, Tykodi SS, Chow LQ, et al.: Safety and activity of anti-PD-L1 antibody in patients with advanced cancer. N Engl J Med 2012;366:2455-2465.

Correspondence: Prof. Dr. Uwe Pleyer, Klinik für Augenheilkunde, Charité Universitätsmedizin Berlin, Campus Virchow-Klinikum, Augustenburger Platz 1, 13353 Berlin, Germany, uwe.pleyer@charite.de 